\title{
Median Nerve Neuritis after Infection by the SARS-CoV-2 Virus
}

\author{
Marcelo de Pinho Teixeira Alves ${ }^{1 *}$ and João Pedro Gouveia Nóbrega ${ }^{2}$ \\ ${ }^{1}$ Department of Surgery, Hospital Dr. José Maria Grande, Portalegre, Portugal \\ ${ }^{2}$ Department of Orthopedic Surgery, Hospital do Espírito Santo, Évora, Portugal
}

\begin{abstract}
Background: The spectrum of neurological complications from SARS-CoV-2 infection is under constant investigation. According to numerous publications, occurrence of neurological complications can be about $36.4 \%$. There are no reports to date of the occurrence of bilateral median nerve neuritis in the wrist after SARS-CoV-2 infection.

Case: A previously healthy 24 years old female was observed in March 2021 due to dysesthesias in both hands, associated with nocturnal discomfort and decreased grip strength. The symptoms started a month earlier with acroparesthesias. SARS-CoV-2 virus infection was in January 2021 and evolved favorably, with progressive remission in about 10 days. Prior to the infection she didn't have any of the hand symptoms; denied diabetes or metabolic diseases. Negative tests for SARS-CoV-2 infection since February. April 2021, the patient returned with persistent dysesthesias in her hands. Normal EMG, however with Sensitive Conduction Speed (SCS) of $50 \mathrm{~m} / \mathrm{sec}$ on the right wrist; on the left SCS $55 \mathrm{~m} / \mathrm{sec}$. Sensitive latency 2.8 on the right and 2.6 on the left. Amplitude 17 on the right and 24 on the left. MRI detected an increase in signal in PDFS sequences in both median nerves, in the carpal tunnel, with no detected variations in cross-sectional areas or masses. The treatment was a 30 days $150 \mathrm{mg}$ benfotiamine in two daily doses.
\end{abstract}

Discussion: There is a wide variation in the clinical presentation of SARS-CoV-2 infection, ranging from asymptomatic patients to death. Most frequent symptoms are fever, dry cough, sore throat, dyspnea, myalgia, fatigue, chills, diarrhea, chest pain and vomiting, and the most common complications pneumonia and acute respiratory distress syndrome. Neurological manifestations of viral infection can occur in about $36.4 \%$ and in $45.5 \%$ in severe disease. They include GuillánBarré and Miller Fischer syndrome; however, these conditions usually occur in more severe cases of the disease, but this patient hadn't the typical picture of ataxia, ophthalmoplegia and areflexia. Benfotiamine is a synthetic derivative of thiamine with a multifaceted therapeutic profile, with a therapeutic role especially in diabetic neuropathy. Marcelo de Pinho Teixeira Alves, Department of Surgery, Orthopedics Service, Hospital Dr. José Maria Grande, Av. de Santo António, 6ªndar, 7300-853, Portalegre, Portugal,

E-mail:dr.marcelo@sapo.pt

Received Date: 19 Jul 2021

Accepted Date: 20 Sep 2021

Published Date: 23 Sep 2021

Citation:

de Pinho Teixeira Alves M, Nóbrega

JPG. Median Nerve Neuritis after Infection by the SARS-CoV-2 Virus.

Clin Surg. 2021; 6: 3306.

Copyright $(2021$ Marcelo de Pinho Teixeira Alves. This is an open access article distributed under the Creative Commons Attribution License, which permits unrestricted use, distribution, and reproduction in any medium, provided the original work is properly

Conclusion: Knowledge and recognition of neurological manifestations of SARS-CoV-2 infection and early detection of secondary peripheral neuropathies result in improvement of patients' clinical results and development of ideal ways of treatment. Although only a small percentage of patients develop peripheral neuropathy, in a pandemic this can result in a major social and health impact. The reported case is the first case of bilateral secondary median nerve neuritis in the literature. Level of evidence 4.

\section{Introduction}

The spectrum of neurological complications from SARS-CoV-2 infection is under constant investigation, especially if we see that the pandemic has reached more than 200 countries globally $[1,2]$. Even with all vaccination procedures worldwide, there are still new infections and complications.

According to numerous publications available [1-5], the occurrence of neurological complications, both central and peripheral, can affect about $36.4 \%$ of patients infected with the SARS-CoV-2 virus (COVID-19). According to Nersesjan et al. [6] neurological manifestations can occur during or after viral infection, but the mechanism by which this occurs and the frequency of their occurrence remains poorly understood.

There are no reports to date of the isolated occurrence of bilateral neuritis of the median nerve in the wrists after infection by the SARS-CoV-2 virus, and this work presents this case. 


\section{Case Presentation}

Previously healthy female patient, 24 years old, observed at the Orthopedics consultation in March 2021 due to complaints of dysesthesias in both hands, especially on the right, associated with nocturnal discomfort and decreased grip strength. She reported having started the picture a month earlier with the emergence of acroparesthesias.

She reported infection with the SARS-CoV-2 virus in January 2021 and had had fever, dyspnea, chest pain, dysgeusia and anosmia. She reported that the SARS-CoV-2 virus infection had evolved favorably, with progressive remission in about 10 days.

She reported that, prior to infection by the SARS-CoV-2 virus; she did not have any of the symptoms reported on the hands. She denied diabetes mellitus or other metabolic diseases.

During the orthopedic consultation, she still reported easy tiredness, without the other complaints related to the infection.

Updated tests for SARS-CoV-2 negative since February. Complementary examinations were requested: Electromyography (EMG) of the upper limbs and study by Magnetic Resonance Imaging (MRI) of both wrists. In April 2021, the patient returned to the consultation with dysesthesias in her hands. Normal EMG, however showing asymmetries. Sensitive conduction speed (VCS) of $50 \mathrm{~m} / \mathrm{sec}$ on the right wrist; on the left VCS $55 \mathrm{~m} / \mathrm{sec}$. Sensitive latency 2.8 on the right and 2.6 on the left. Amplitude 17 on the right and 24 on the left.

The MRI study detected an increase in signal in Proton Density with Fat Suppression (DPFS) in both median nerves, located in the carpal tunnel segment, without detecting abrupt variations in their gauges or lesions occupying adjacent space (Figure 1).

The treatment initially instituted was expectant, associating rest and prescription of $150 \mathrm{mg}$ benfotiamine in two daily doses, for 30 days.

\section{Discussion}

There is a wide variation in the clinical presentation of SARS-
CoV-2 virus infection, with different degrees of severity, ranging from asymptomatic patients to fatal cases. The most frequent symptoms of the infection are fever, dry cough, sore throat, dyspnea, myalgia, fatigue, chills, diarrhea, chest pain and vomiting, with the most common complications being pneumonia and acute respiratory distress syndrome $[2,7]$.

Reports from Wuhan, China, showed that neurological manifestations of viral infection can occur in about $36.4 \%$ of patients and in $45.5 \%$ of patients with severe disease [3]. In the study by Nersesjan et al. [6] muscle weakness was the most common neurological symptom (34.4\% of patients), being much more common in critically ill patients (54\%) and admitted to Intensive Care Units (ICU) than in patients who did not need it of ICU (7.7\%). However, in their study, they did not detect the occurrence of peripheral neuropathies in the upper limbs.

According to the current literature, a large spectrum of peripheral neurological manifestations is observed in the infection by the SARSCoV-2 virus, including Guillán-Barré syndrome and Miller Fischer syndrome [8]. Guillán-Barré syndrome and its variants (MillerFischer syndrome) represent $21 \%$ of all case reports [7,9]. These conditions usually occur in more severe cases of the disease, which was not the case of the patient presented, who did not have the typical condition of ataxia, ophthalmoplegia and areflexia [10]. The patient in question presented, during the course of the viral infection, the most common symptoms of involvement of the peripheral nervous system - anosmia and ageusia, being within the usual series of COVID-19. This fact is confirmed in several literature reviews on the neurological manifestations and complications of COVID-19, which detected ageusia or hypogeusia, hyposmia, neuralgia, muscular atrophy and Guillán-Barré syndrome as the most common peripheral nervous system complications $[11,12]$.

A case of peripheral neuropathy in the lower limbs associated with infection by SARS-CoV-2 viruses has recently been reported [13] This case differs from the case of the patient presented by two factors - being affected by the lower limbs and having started unrelated to respiratory involvement, which started seven days after the onset of the neurological condition.
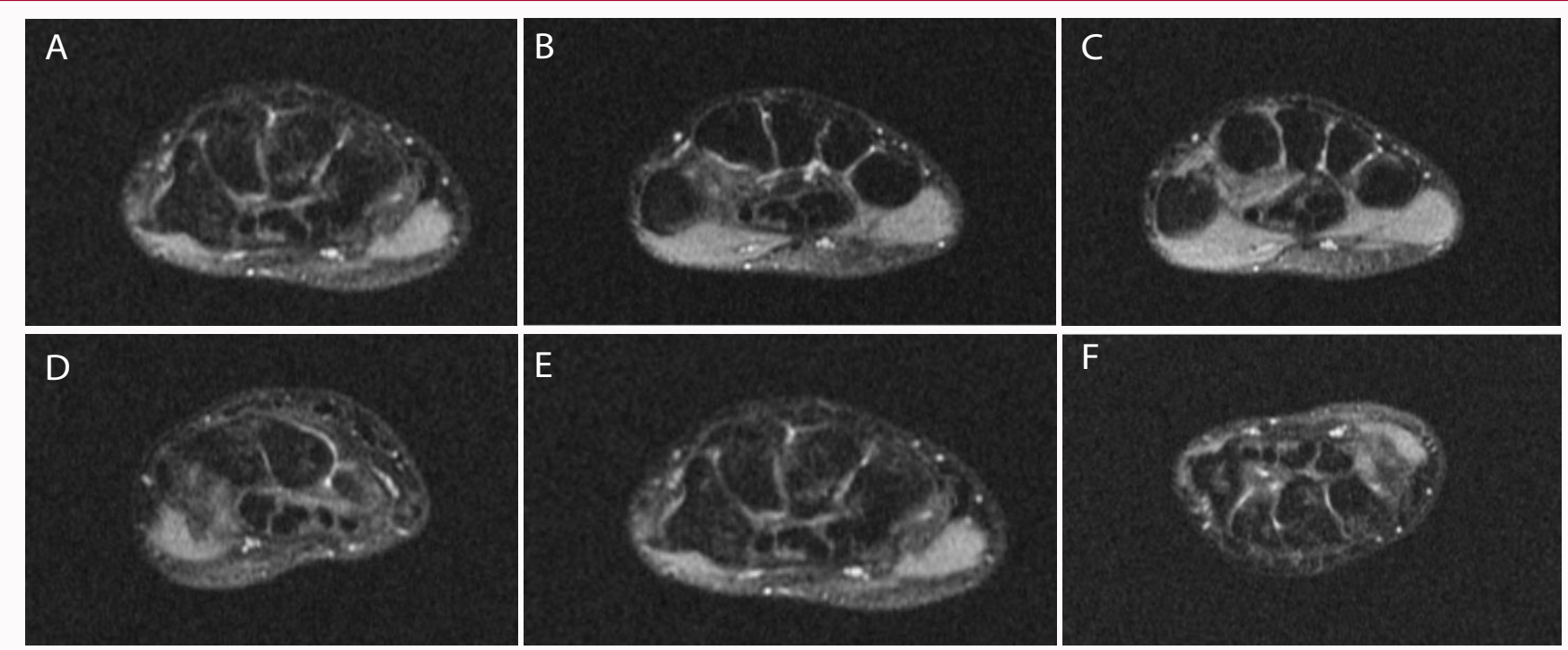

Figures 1: A-F) Magnetic Resonance images displaying increase in signal in Proton Density with Fat Suppression (DPFS) in both median nerves (A-D: Right hand; E-F: Left hand), located in the carpal tunnel segment, without detecting abrupt variations in their gauges or lesions occupying adjacent space. 
The case of the reported patient is, until now, unique in the literature. In addition to the clinical diagnosis of peripheral neuropathy secondary to COVID-19, electrophysiological examinations are commonly nonspecific. Often difficult to perform due to the clinical condition of patients and the ICU stay for a prolonged period. Daia et al. [14] underwent electrophysiological examination in three patients who had myalgia and fatigue in both lower limbs. They detected the presence of conduction block in several nerves, suggesting demyelinating polyneuropathy related to COVID-19. The patient's EMG was normal, despite showing discrepancies between the tested limbs.

The imaging of peripheral nerves assists clinical diagnosis and can guide treatment in patients with COVID-19 with neuromuscular symptoms that arise from viral infection, from the course of hospitalization or secondarily to treatment. Advanced imaging techniques such as nuclear magnetic resonance neurography are well-established tools for assessing peripheral nerve damage [15]. High-resolution resonance of the peripheral nerve can detect macro or microstructural changes in the nerve [15-18].

Significant findings in MRI studies of peripheral nerves include hyper-intensity of signal in fluid-sensitive sequences, changes in nerve caliber, architectural distortion and nerve discontinuity, effects of extrinsic mass and compression [15]. In the patient presented, an increase in the signal was observed in the proton density and fat suppression sequences, which are sensitive to fluids; however, no other changes were observed such as those described above. In the presence of the normal EMG study, MRI was extremely valuable in determining the involvement of the median nerve in the carpal tunnel, being able to accurately locate the pathology and determine the extent of the lesion [15].

Paterson et al. [5] observed in their study a patient who developed brachial plexopathy (Parsonage-Turner syndrome) two weeks after the onset of COVID-19. The treatment instituted was corticotherapy. These authors reported that the patient was recovering when his work was published. The treatment instituted for this patient initially was $150 \mathrm{mg}$ benfotiamine in two daily doses. Benfotiamine is a synthetic derivative of thiamine that has a multifaceted therapeutic profile, having been shown to have a therapeutic role especially in neuropathies secondary to diabetes [19-21], being effective in reducing neuropathic pain. This treatment must be individualized, based on the characteristics of the patients to be treated. The patient is currently recovering.

\section{Conclusions}

The knowledge and recognition of the neurological manifestations of the SARS-CoV-2 virus infection and the early detection of associated secondary peripheral neuropathies results in the improvement of the patients' clinical results and the development of ideal forms of treatment.

Although only a small percentage of patients develop peripheral neurological symptoms, in a pandemic situation this can result in a major social and health impact. The reported case is the first case of neuritis secondary to bilateral COVID-19 of the median nerve reported in the literature. Clinical diagnosis is essential and magnetic resonance imaging can confirm the injury to the peripheral nerve.

Further future studies are extremely necessary to understand the pathophysiology of peripheral neurological damage secondary to infection by the SARS-CoV-2 virus.

\section{References}

1. Bureau BL, Obeidat A, Dhariwal MS. Peripheral neuropathy as a complication of SARS-CoV-2. Cureus. 2020;12(11):e11452.

2. Baj J, Karakula-Juchnowicz H, Teresinski G, Buszewicz G, Ciesielka M, Sitarz E, et al. COVID-19: Specific and non-specific clinical manifestations and symptoms: The current state of knowledge. J Clin Med. 2020;9(6):1753.

3. Ftiha F, Shalom M, Jradeh H. Neurological symptoms due to Coronavirurs disease 2019. Neurol Int. 2020;12(1):8639.

4. Ellul MA, Benjamin L, Singh B, Lant S, Michael BD, Easton A, et al. Neurological associations of COVID-19. Lancet Neurol. 2020;19(9):76783.

5. Paterson RW, Brown R, Benjamin L, Nortley R, Wiethoff S, Bharucha T et al. The emerging spectrum of COVID-19 neurology: Clinical, radiological and laboratory findings. Brain. 2020;143(10):3104-20.

6. Nersesjan V, Amiri M, Lebech AM, Roed C, Mens H, Russell L, et al. Central and peripheral nervous system complications of COVID-19: A prospective tertiary center cohort with 3-month follow-up. J Neurol. 2021;268:3086-104.

7. Niazkar HR, Zibaee B, Nasimi A, Bahri N. The neurological manifestations of COVID-19: A review article. Neurol Sci. 2020;41:1667-71.

8. Chen X, Laurent S, Onur OA, Kleineberg NN, Fink GR, Schweitzer F, et al. A systematic review of neurological symptoms and complications of COVID-19. J Neurol. 2021;268(2):392-402.

9. Maury A, Lyoubi A, Peiffer-Smadja N, de Broucker T, Meppiel E. Neurological manifestations associated with SARS-CoV-2 and other coronaviruses: A narrative review for clinicians. Revue Neurol(Paris). 2021;177(1-2):51-64.

10. Gupta SK, Jha KK, Chalati MD, Alashi LT. Miller Fischer syndrome. BMJ Case Rep. 2016;2016:bcr2016217085.

11. Ahmad I, Rathore FA. Neurological manifestations and complications of COVID-19: A literature review. J Clin Neurosci. 2020;77:8-12.

12. Nepal G, Rehrig JH, Shresha GS, Shing YK, Yadav JK, Ojha R, et al. Neurological manifestations of COVID-19: A systematic review. Critical Care. 2020;24(1):421.

13. Abdelnour L, Eltahir Abdalla M, Babiker S. COVID 19 infection presenting as motor peripheral neuropathy. J Formos Med Assoc. 2020;119(1):111920.

14. Daia C, Scheau C, Neagu G, Andone I, Spanu A, Popescu C, et al. Nerve conduction study and electromyography findings in patients recovering from COVID-19 - Case report. Int J Inf Dis. 2021;103:420-22.

15. Fernandez CE, Franz CK, Ko JH, Walter JM, Koralnik IJ, Ahlawat S, et al. Imaging review of peripheral nerve injuries in patients with COVID-19. Radiology. 2020;298(3):E117-E130.

16. Deshmukh S, Carrino JA, Feinberg JH, Wolfe SW, Eagle S, Sneag DB. Pins and needles from fingers to toes: high resolution MRI of peripheral sensory mononeuropathies. Am J Roentgenol. 2017;208(1):W1-w10.

17. Deshmukh SD, Samet J, Fayad LM, Ahlawat S. Magnetic resonance neurography of traumatic pediatric peripheral nerve injury: Beyond birthrelated brachial palsy. Pediatr Radiol. 2019;49(7):954-64.

18. Deshmukh S, Fayad LM, Ahlawat S. MR Neurography (MRN) of the long thoracic nerve: Retrospective review of clinical findings and imaging results at our institution over 4 years. Skeletal Radiol. 2017;46(11):1531-40.

19. Sheng L, Cao W, Lin P, Chen W, Xu H, Zhong C, et al. Safety, tolerability and pharmacokinetics of single and multiple ascending doses of benfotiamine in healthy subjects. Dru Des Devel Ther. 2021;15:1101-10. 
20. Ziegler D, Papanas N, Schnell O, Nguyen BDT, Nguyen KT, Kulkantrakorn $\mathrm{K}$, et al. Current concepts in the management of diabetic polyneuropathy. J Diabetes Investig. 2021;12(4):464-75.
21. Haupt E, Ledermann H, Köpcke W. Benfotiamine in the treatment of diabetic polyneuropathy -- a three week-randomized controlled pilot study (BEDIP study). Int J Clin Pharmacol Ther. 2005;43(2):71-7. 